\title{
Médiévales
}

Langues, Textes, Histoire

55 | automne 2008

Usages de la Bible

\section{Don Quichotte et « nuestro romance castellano »: un écho tardif du combat médiéval pour la langue} romane

Don Quijote and "Nuestro Romance Castellano", a Belated Echo of the Medieval Fight for the Romance Language

\section{Pierrette Pellen-Barde}

\section{OpenEdition}

Journals

Édition électronique

URL : http://journals.openedition.org/medievales/5485

DOI : $10.4000 /$ medievales. 5485

ISSN : 1777-5892

Éditeur

Presses universitaires de Vincennes

Édition imprimée

Date de publication : 20 décembre 2008

Pagination : 135-145

ISBN : 978-2-84292-221-4

ISSN : 0751-2708

\section{Référence électronique}

Pierrette Pellen-Barde, «Don Quichotte et « nuestro romance castellano » : un écho tardif du combat médiéval pour la langue romane », Médiévales [En ligne], 55 | automne 2008, mis en ligne le 02 novembre 2010, consulté le 19 avril 2019. URL : http://journals.openedition.org/medievales/5485 ; DOI : 10.4000/medievales.5485

Ce document a été généré automatiquement le 19 avril 2019.

Tous droits réservés 


\title{
Don Quichotte et « nuestro romance castellano » : un écho tardif du combat médiéval pour la langue romane
}

\author{
Don Quijote and "Nuestro Romance Castellano", a Belated Echo of the Medieval \\ Fight for the Romance Language
}

Pierrette Pellen-Barde

1 «Tous les poètes de l'Antiquité écrivirent dans la langue qu'ils tétèrent avec le lait... " ", déclare don Quichotte à don Diego de Miranda, le Caballero del Verde Gabán, dont le fils, tout nourri de Virgile et d'Homère, dédaigne la "poesía de romance ", c'est-à-dire la poésie en langue romane. Ici se dessine un thème récurrent dans l'œuvre, mais que l'on pourrait croire obsolète au Siècle d'or, l'affirmation de la dignité de la langue romane.

Or, Cervantès feint de ne pas être l'auteur du Quichotte dans cette «langue tétée avec le lait ", et prétend nous faire croire à la fiction d'un «primer autor» en langue arabe. Aucune nouveauté là-dedans, les auteurs des premiers romans français en prose, au XIII ${ }^{\mathrm{e}}$ siècle, et leur postérité, ayant eux aussi tendu à faire passer leurs œuvres pour des traductions d'originaux écrits dans une langue ancienne prestigieuse ${ }^{2}$. Il est vrai que si la littérature vernaculaire en vers avait été la première à conquérir, grâce à une longue étape d'expérimentation orale, une légitimité, celle en prose avait tardé à s'imposer, la prose vernaculaire étant jusque-là l'apanage de textes soi-disant véridiques, textes religieux, juridiques, ou annales. Entrait aussi en cela le vieux complexe d'infériorité linguistique des langues vulgaires envers le grec et le latin - « reinas de las lenguas ", selon don Quichotte, face à ces «lenguas fáciles » que sont les langues modernes ${ }^{3}$ - et envers d'autres langues aussi prestigieuses que l'arabe ou l'hébreu.4. Mais grâce à la fiction d'un original en langue ancienne pour se justifier d'écrire en prose romane, on avait bel et bien lancé un mouvement qui allait faire des premiers romans français de formidables instruments de promotion de la langue vernaculaire. 
3 La Castille connut un parcours similaire, mais plus tardif: les premières créations littéraires en prose y ayant été, au tournant du XIV siècle, adaptées de la production d'outre-Pyrénées, elles s'inscrivirent dans cette tradition ennoblissante de l'auteur fictif antérieur, tradition que tendirent à reprendre aussi les œuvres originales castillanes. Le Libro del Caballero Zifar, vers 1300, se prétend une traduction du chaldéen, et Montalvo invente deux cents ans plus tard un soi-disant original grec à ses Sergas de Esplandián. Et cet original aurait été découvert dans les fondations d'un ermitage, trait qu'il est curieux de voir réapparaitre dans le Quichotte, à la fin de la première partie, à propos de la suite de l'œuvre. Revenons donc à Cervantès, qui a parodié, en son temps, la tradition de l'auteur fictif en inventant Cide Hamete Benengeli, "primer autor árabe» dont il aurait fait traduire l'œuvre intégrale en "romance». Recourir au subterfuge de la traduction impliquait l'anachronisme d'endosser le complexe médiéval des premières fictions en prose romane. Celui du péché de fantaisie, l'œuvre étant occultée derrière un travail historiographique où se rejoignent, selon un montage ambigu, Cide Hamete, « historien très méticuleux et très exact ", "chroniqueur de cette grande histoire $»^{5}$, et Cervantès, explorateur d'archives manchoises. Or, même si les deux auteurs garantissent l'autorité d'une histoire qualifiée avec insistance de "verdadera", "vraie», en se retranchant derrière archives, annales et chroniques, le Quichotte, comme les romans de chevalerie qu'il parodie, est aussi "plein de folies ${ }^{6}$ » que le dit le curé de ceux de l'aubergiste. Car, même si Cervantès est partagé entre la défense de la vérité ${ }^{7}$ et l'amour du romanesque, c'est semble-t-il ce dernier qui l'emporte dans le Quichotte, faisant pièce, à la façon des premiers romans en prose, au vieux complexe de la prose castillane originelle de fiction. Ainsi se trouvent réactualisées les étapes d'un processus où la reconnaissance de celle-ci avait été postérieure à la reconnaissance de la poésie en castillan: «[...] l'épique peut s'écrire aussi bien en prose qu'en vers ${ }^{8} "$, dit le chanoine, tandis que le curé voit un enrichissement de «nuestra lengua» dans divers préceptes esthétiques par lesquels les auteurs de romans de chevalerie pourraient «devenir aussi fameux en prose que le sont en vers les deux princes de la poésie grecque et de la poésie latine ${ }^{9}$ ".

4 D'autre part, en parodiant des romans de chevalerie soi-disant traduits d'une langue plus ancienne, Cervantès réédite un des processus qui imposèrent la langue romane. Car, en plaçant entre le More Cide Hamete Benengeli et lui-même un "traductor" ou « intérprete », parent littéraire du «traslaudador » - «traducteur » - du Zifar, Cervantès renvoie à l'effervescence traductrice qui anima pendant des siècles la Castille médiévale et connut sa véritable expansion avec l'apparition de l'école des traducteurs de Tolède au douzième siècle ${ }^{10}$. Peu d'hommes maitrisant à la fois l'arabe (ou l'hébreu) et le latin, on procédait selon une première traduction des textes orientaux au « romance »- donc au roman, simple pont linguistique, mais qui engrangea un progrès latent au contact de langues aussi élaborées que ces trois langues -, puis du "romance» au latin, encore langue de culture au $\mathrm{XII}^{\mathrm{e}}$ siècle. À ce système en deux étapes renvoie le Zifar, selon la variante du passage successif du chaldéen au latin, puis - Ferrand Martínez procédant à une intéressante inversion de l'ordre chronologique entre le latin et le "romance » par rapport aux traducteurs tolédans $\mathrm{du} \mathrm{XII}^{\mathrm{e}}$ siècle - du latin au « romance ».

5 Cervantès renvoie, lui, au système le plus répandu chez les traducteurs du siècle suivant: sans étape intermédiaire, avec passage direct de l'arabe au castillan, devenu presque toujours maintenant la langue d'aboutissement de la traduction, système illustré par le Calila et Dimna que fit traduire Alphonse $\mathrm{X}$ au milieu du XIII ${ }^{e}$ siècle. On voit donc Cervantès, double d'Alphonse X, s'amuser à faire du Quichotte une immense version de l'arabe au 
castillan. A « nuestra lengua castellana », ou " nuestro romance castellano », comme le dit don Quichotte ${ }^{11}$, écho au "romance » loué par Juan Ruiz dans le Libro de Buen Amor et au " romance paladino », c'est-à-dire « la langue romane claire, pure », chère à Berceo dans son introduction à la Vida de Santo Domingo de Silos. Cervantès va jusqu'à une mise en abyme de ce phénomène à l'intérieur même des récits imbriqués dans le Quichotte, avec le «cautivo »- «captif »-, qui trouve un renégat parfaitement bilingue pour traduire intégralement de la « lengua arábiga » au "romance » les missives de Zoraida, et avec la soi-disant Micomicona évoquant le texte indéchiffrable, chaldéen à l'instar du Zifar, ou grec à l'instar des Sergas de Esplandián, de la prophétie paternelle. Et Cervantès s'amuse à parodier le schéma en deux étapes de l'école tolédane du XII siècle en prêtant au royaume de Candaya d'où viendrait la Trifaldi une "lengua candayesca », étape intermédiaire entre un parchemin en syriaque et sa traduction définitive en castillan.

Quels que soient les exemples donnés ci-dessus, la langue d'aboutissement s'avère être toujours le castillan. Ainsi, en réactualisant les vieux circuits médiévaux de traduction qui avaient abouti à la consécration du "romance castellano ", c'est à celui-ci que Cervantès donne la vedette. Il est certes loin d'être le premier, mais, écho à la mise en perspective «médiévale » de son œuvre, il semble ressusciter les temps lointains où le castillan s'était imposé comme langue cible. Et, en même temps que sa passion pour la langue romane, il met en relief sa fascination pour le phénomène de la traduction, qu'il montre toujours actuel dans le Quichotte, où se multiplient les références à des œuvres étrangères contemporaines traduites du français ou de l'italien, donc d'une langue romane à une autre langue romane à présent ${ }^{12}$. Fascination qui ressort aussi de nombreuses traductions sur le vif de mots latins, arabes, ou italiens. Le Quichotte lui-même était promis à de multiples traductions dans toutes les langues, ainsi que le prophétise Sansón Carrasco au sujet de la première partie, chose déjà vérifiée dans les versions anglaise et française de 1612 et 1614.

7 Et, chose déjà réalisée « virtuellement » dans la présumée traduction de l'original de Cide Hamete Benengeli par un Morisque «aljamiado », la «aljamía » étant le nom donné au castillan parlé par les Arabes ${ }^{13}$. En quoi transparaît un autre schéma médiéval amplement pratiqué jusqu'au triomphe de la Reconquête sous les Rois Catholiques, celui des contacts entre chrétiens et Mores. Schéma illustré, anachroniquement, par Cide Hamete évoquant au chapitre XLIV de la seconde partie la « comunicación » que lui-même a eue avec des chrétiens, et développé dans les épisodes du "cautivo», de Ricote le Morisque, et des galères. Un contact impliquant lui aussi la traduction, thème décidément incontournable du Quichotte, par exemple entre le « cautivo » et Zoraida. Un contact qui ne se suffit pas de la langue des signes par laquelle s'ouvre la relation entre ces derniers, ni du sabir parlé autour de la Méditerranée, "mezcla de lenguas ", "mélange de langues », et même «bastarda lengua» selon le «cautivo », à l'inverse de la «aljamía» et de la «lengua arábiga » de Zoraida ou de la "lengua morisca» de Ricote. Deux langues que distingue soigneusement don Quichotte au chapitre LXVII de la seconde partie ${ }^{14}$.

8 Ainsi se dessine de plus en plus l'intérêt de don Quichotte, et de Cervantès par le truchement de son héros, pour les langues modernes. Et cela est en outre confirmé par le fait que, dans cette Espagne du XVII ${ }^{e}$ siècle qui se ferme à l'Europe, le Quichotte montre une curiosité affable envers les autres langues, d'où cet éventail linguistique extrêmement ouvert, où figurent non seulement les langues anciennes, ou encore l'arabe, toujours vivant, voire la « lengua chinesca », le chinois, dans un trait d'humour de la Dedicatoria al 
conde de Lemos, mais aussi un généreux appel de celles parlées dans l'Europe contemporaine ${ }^{15}$.

Et les langues anciennes? Don Quichotte, qui voit en celles-ci «le premier échelon des sciences ${ }^{16}$ ", établit lors de sa visite à l'imprimerie barcelonaise une intéressante distinction entre le latin et le grec, «reinas de las lenguas » - qu'il ne voit point comme des langues mortes -, et les langues modernes, « lenguas fáciles ». Un jugement de valeur donc, au profit des premières, peut-être motivé par le terreau que constituent toujours langues et cultures anciennes. Ce qui transparaît dans la définition par notre héros de ces "modernos romancistas", "auteurs modernes en langue romane ", dédaignés par un don Lorenzo de Miranda rétif à la "poesía de romance »: "[...] ils ignorent d'autres langues et d'autres sciences qui orneraient, éveilleraient et soutiendraient leur élan nature ${ }^{17}$ ». Des « romancistas » qui se privent donc de la sève d'une culture classique toujours vivante et abondamment citée dans le Quichotte, non reniée par le héros luimême, et à laquelle s'est abreuvé don Lorenzo pendant ses six années d'études à Salamanque. Une culture également lue dans la langue d'origine par le père de celui-ci, don Diego de Miranda, dont la bibliothèque, réconciliant langues ancienne et moderne, contient des ouvrages « soit en langue romane, soit en latin ${ }^{18}$ ».

10 Mais les langues anciennes sont parfois affectées d'une coloration négative liée à une propension au style recherché, par exemple dans des latinismes, tel "ínclito", « illustre ", ou des alternatives étymologiques élégantes ${ }^{19}$, ou de pédantes citations latines ${ }^{20}$, souvent minées par un contexte comique auquel concourt Sancho Panza. D'où une perspective dévalorisée, soulignée par le terme péjoratif « latinicos» dont l'ami consulté dans le Prologue qualifie le fatras auquel il déconseille à Cervantès d'avoir recours, et par les métaphores de la «jerigonza», «jargon $»^{21}$, ou le syntagme «mal latín» par lequel don Lorenzo caractérise une erreur. Car pour Cervantès, même s'il reconnaît le pouvoir d'enrichissement des langues et de la culture anciennes, il est patent que l'époque à laquelle renvoient ces langues et cette culture est "pretérita ", selon les propres termes de don Quichotte ${ }^{22}$. Et celui-ci, qui ne devient clairvoyant qu'à la fin du roman quant à ses illusions chevaleresques, l'a toujours été quant à la prééminence, depuis longtemps affirmée, de la langue romane sur le latin. Berceo, qui, au xiII siècle, dans la strophe 609 de la Vida de Santo Domingo de Silos, évoque un parchemin illisible en "cerrado latino", "latin hermétique", ne s'est pas trompé lui non plus sur ce latin devenu incompréhensible. Pas plus que le roman de chevalerie espagnol, adapté du français, autre langue romane, ou écrit directement en castillan, et qui, dans Amadís, définit comme "muy escuro" le latin inscrit sur la poitrine du petit Esplandián, latin qu'il convient d'opposer au « lenguaje de Alemaña y otras tierras » ajouté à la culture classique de son père.

11 Voilà donc Cervantès et don Quichotte champions de la langue romane, et ce d'autant plus que l'œuvre semble s'inscrire face à un courant de pédanterie latinisante, que laissent entrevoir les termes par lesquels l'ami de l'auteur conclut dans le Prologue un chapelet de formules latines: «[...] avec ces latinismes savantasses [...] vous passerez à tout le moins pour grammairien, source aujourd'hui du plus grand honneur et du plus grand profit $»^{23}$. Et champions de la vitalité de la langue romane, vitalité entretenue par « le vulgaire et l'usage », selon une formule de don Quichotte dans ses préceptes à Sancho futur gouverneur. Cette « langue que commandent le vulgaire et l'usage $»^{24}$ ne peut être que la langue parlée, dont on notera la variété expressive des termes la désignant dans le 
Quichotte : «romance », qu'employaient au Moyen Âge Berceo ou Juan Ruiz ${ }^{25}$, « romance castellano », « lengua castellana », « vulgar castellano », « castellano » ${ }^{26}$.

N'oublions pas que, ancien captif, Cervantès sait mieux que tout autre, à l'instar du «cautivo » et de Ricote le Morisque - l'expulsé revenu en Espagne -, que la langue maternelle charrie comme du sang tout ce qui fait la vie profonde de la patrie perdue. Et ce Saavedra cité dans le roman pourrait reprendre à son compte la profession de foi de Ricote, plus mal traité dans la Berbería où il a été expulsé que dans son Espagne natale : «[...] le désir que nous avons tous de revenir en Espagne est si grand que la plupart de ceux (et ils sont nombreux) qui savent la langue comme moi y reviennent [...] laissant làbas femmes et enfants [...] car il est doux l'amour de la patrie $»^{27}$. Ainsi est mis en avant le lien très fort entre langue et patrie. Et c'est Ricote, pourtant morisque, dont la langue est la "pura castellana" nullement mâtinée de sa langue morisque, qui exprime cette relation avec le plus de vigueur. Affirmation reprise en termes voisins par sa fille Ana Félix, qui pour rejeter son héritage morisque se prévaut de sa «langue», ses "bons principes", et sa "foi catholique » dont elle dit, reprenant les propres termes de don Quichotte sur la langue maternelle, qu'elle l'a "tétée avec le lait ${ }^{28} »$. Il est d'ailleurs significatif que Cervantès emploie le raccourci « elle ne savait pas parler chrétien ${ }^{29}$ à propos de Zoraida, jeune More à l'impétueux désir d'intégration catholico-hispanique. Voilà donc souligné le lien entre la langue et les valeurs fondatrices - amour de la patrie, foi, culture - qu'elle véhicule, assimilation valable seulement pour les langues vivantes. $\mathrm{Ne}$ vaut également que pour ces dernières l'insistance cervantine à assigner un tendre possessif, adjectif ou pronom, à l'énoncé des diverses langues parlées dans le Quichotte, à commencer dans la bouche du héros évoquant "nuestro romance castellano » ou «nuestra lengua castellana $~_{30}$. Ce qui rejette encore plus le latin, exclu du possessif, dans une époque "pretérita ", et souligne le lien charnel entre la langue maternelle et celui qui la parle ${ }^{31}$.

13 Une langue qui avait été vue plus tôt qu'ailleurs en Europe comme fondant la nation, notamment dans les actes juridiques de deux grands rois du XIII ${ }^{e}$ siècle, Ferdinand III et, plus encore, son fils Alphonse X. Et une langue reconnue dans ses capacités à accueillir le savoir, en particulier à partir de ce même Alphonse X. Lequel s'était démarqué parmi les souverains de l'Europe médiévale non seulement par son œuvre encyclopédique, mais aussi par sa volonté de répandre le savoir en langue romane. Cet amour alphonsin du savoir anime aussi Cervantès et don Quichotte. Ce dernier, qui voit dans les langues anciennes « le premier échelon des sciences », a accédé, au moins en partie, à cet échelon, lui qu'on voit avoir des connaissances en latin ${ }^{32}$. Et cet autodidacte inspiré, ouvert aux autres langues parlées, par exemple à l'italien ${ }^{33}$, et à l'arabe, sans préjugés, à une époque où l'Espagne se replie sur elle-même, montre qu'il a accédé à d'autres échelons, dont le domaine littéraire, la plume étant pour lui selon une métaphore rattachée elle aussi au champ linguistique "langue de l'âme ${ }^{34}$ ». Un domaine littéraire non limité au chevaleresque, et illustré par ces citations dont grouille le texte du Quichotte et par l'évocation des plus grands noms des littératures castillane et européenne. Et, dans un prodigieux effet de miroir, un domaine littéraire où don Quichotte et Sancho Panza voient figurer la première partie de leurs propres aventures.

Or, pour arriver au chef-d'œuvre de Cervantès, il avait fallu, couronnés par la Renaissance et le Siècle d'Or, les longs siècles médiévaux au cours desquels avait reculé - et Cervantès, "père » et non «parâtre » quoi qu'il en dise ${ }^{35}$, ne relâche pas sa vigilance, comme si le combat était toujours d'actualité ${ }^{36}$ - le latin face au roman, et s'étaient aiguisées les 
possibilités expressives de la «lengua romance ». L'énoncé de celles-ci est patent dans l'œuvre, par exemple dans le rejet, par l'ami consulté dans le Prologue, des genres latinisants et « latinicos » d'emprunt au profit de « mots expressifs [...] et bien trouvés » qui, eux, ne peuvent être qu'en castillan, et donc dans la « langue tétée avec le lait ${ }^{37}$. Un castillan que domine Cervantès, comme don Quichotte, précepteur de «buen romance " pour Sancho Panza, ce dernier dût-il voir en son maître un «censeur de vocables $\aleph^{38}$. Nous ne nous attarderons pas sur la variété des registres et la virtuosité linguistique de Cervantès, dont chaque page révèle l'immense plaisir qu'il retire de l'écriture et du jeu sur la langue romane. Insistons seulement sur le fait que la langue de don Quichotte devient de plus en plus moderne au fur et à mesure que le héros retrouve sa lucidité, et que Cervantès, si on le compare à d'autres auteurs du Siècle d'Or, se révèle déjà - à part quelques traits morphologiques et syntaxiques aujourd'hui disparus - un auteur extrêmement moderne. Cela confirme bien à quel point, écho lointain au long processus par lequel le roman en prose avait conquis ses lettres de noblesse, Cervantès avait su «devenir fameux en prose», comme le dirait le curé, et ajoutons : « en prose castillane ».

Malraux fait dire à Magnin, l'un des volontaires français de L'Espoir, « qu'il n'avait jamais pensé que don Quichotte eût été jeune ». Mais qui dans ce roman - où Cervantès réactive le vieux schéma médiéval des traductions comme pour nous rappeler le chemin parcouru par la langue et la prose en « romance »-, qui, en dehors de notre héros, se montre assez moderne pour prôner la primauté de la langue romane sur les langues anciennes, fussentelles « reinas de las lenguas » et véhicules d'une culture toujours vivante? Et pour, une fois débarrassé de son fatras chevaleresque, parler une langue déjà aussi proche de nous, captée dans ce mouvement continu qu'entretiennent «le vulgaire et l'usage», et qui ouvrit un champ immense à une littérature laïque castillane à laquelle Cervantès imprime un tournant décisif. Une langue que don Quichotte appelle tendrement «nuestro romance castellano ", comme dans ce lointain Moyen Âge où la langue romane - nommée «nuestro lenguage de España » dans le Setenario d'Alphonse X - mena un long combat pour s'imposer. Un combat gagné depuis longtemps, mais que l'on croirait encore présent à l'esprit de Cervantès, fût-ce contre certaine pédanterie latinisante, et dont notre héros, porte-parole de l'auteur, est le champion sans reproche.

\section{NOTES}

1. miguel De Cervantes, Don Quijote de la Mancha, J. J. Allen (éd.), Madrid, 2005, seconde partie, XVI, p. 160 : [...] todos los poetas antiguos escribieron en la lengua que mamaron en la leche [...]». Suite du texte : «[...] ils n'allèrent pas chercher dans les langues étrangères de quoi exprimer leurs concepts élevés. C'est donc avec raison [...] que l'on ne devrait pas mépriser le poète allemand parce qu'il écrit dans sa langue, ni le Castillan, ni même le Biscaïen, qui écrit dans la sienne » («[...]y no fueron a buscar las estranjeras para declarar la alteza de sus conceptos. Y siendo esto así, razón sería [...] que no se desestimase el poeta alemán porque escribe en su lengua, ni el castellano, ni aún el vizcaíno, que escribe en la suya »). 
2. En quoi ils singeaient une particularité propre aux premiers romans - au sens étymologique de «mises en langue romane "- de la France du XII ${ }^{\mathrm{e}}$ siècle, lesquels, tel le Roman de Thèbes, avaient été d'authentiques adaptations en vers français de récits poétiques latins.

3. Seconde partie, LXII, p. 554. En Castille comme en France, la poésie romane - épique ou lyrique, sans oublier les premiers romans arthuriens français-précéda la prose romane littéraire. Le "complexe" de la prose romane face au latin ne disparut en Castille que dans la seconde moitié du $\mathrm{XIII}^{\mathrm{e}}$ siècle pour l'historiographie - avec les deux chroniques castillanes d'Alphonse $\mathrm{X}$ - et au tournant du XIV e siècle pour la prose littéraire, avec les traductions de la Vulgate et de la Post-Vulgate, elles-mêmes du XIII ${ }^{\mathrm{e}}$ siècle. N'oublions pas que, bien après Cervantès, les textes savants, tels ceux de Spinoza ou de Newton, furent imprimés en latin, le Saint-Office reprochant à Galilée d'avoir écrit en italien son Dialogo sur les deux systèmes du monde. Les déclarations d'amour de Cervantès à la langue romane s'inscrivent contre un latin qui n'est pas totalement une langue morte.

4. Cervantès voit dans l'hébreu « une autre langue, meilleure et plus ancienne » (« otra mejor y más antigua lengua », première partie, IX, p. 179). Ces langues, pour la plupart véhicules de la parole sacrée, sont en outre définies comme hermétiques : « je ne sais pas les lire » (« yo no las sé leer »), dit par exemple Micomicona des lettres chaldéennes ou grecques de la soi-disant prophétie paternelle (première partie, XXX, p. 422).

5. « [...] historiador muy curioso y muy puntual» (première partie, XVI, p. 241), « coronista desta grande historia » (seconde partie, XXVII, p. 260).

6. «[...] llenos de disparates» (première partie, XXXII, p.443).

7. Il pointe l'erreur de l'aubergiste, qui croit ses romans vrais, étant imprimés, « avec permission de ces messieurs du Conseil royal » (« con licencia de los señores del Consejo Real », première partie, XXXII, p. 445).

8. «[...] la épica también puede escribirse en prosa como en verso.» (première partie, XLVII, p. 621).

9. «[...] hacerse famosos en prosa, como lo son en verso los dos príncipes de la poesía griega y latina » (première partie, XLVIII, p. 622).

10. Maints textes antiques emportés dans leur exil à Damas et Bagdad par des Juifs et des chrétiens hérétiques réfugiés de l'Empire d'Orient et qui, traduits en arabe, suivirent l'expansion musulmane au Maghreb et en Espagne, furent retrouvés à Tolède à côté d'originaux arabes et hébreux après la reconquête de la ville en 1085.

11. Première partie, XIII, p. 209 et seconde partie, LXVII, p. 589. « Nuestra lengua », dit Cervantès lui-même (seconde partie, Dedicatoria, p. 30).

12. Mais, bien qu'il l'appelle "exercice digne de louange ", "loable ejercicio ", don Quichotte émet dans l'imprimerie barcelonaise des réserves sur la traduction, son résultat lui faisant l'effet d'une tapisserie vue à l'envers. Défaut qu'il ne pointe pas pour les langues anciennes - en raison peut-être de leur passage impératif à la traduction-, mais qu'il souligne pour les langues modernes (seconde partie, LXII, p. 554). Une imprimerie a déjà été évoquée (seconde partie, XXII, p. 213), ce qui n'est peut-être pas fortuit, l'imprimerie ayant été un formidable instrument de promotion des langues vulgaires - Amadís de Gaula fut imprimé en 1508 -, même si les premiers ouvrages imprimés, Vulgate en tête, le furent en latin.

13. Dits alors « ladinos ", tel le père de Zoraida (première partie, XLI, p. 549). Le terme « ladino » rappelle l'origine latine du castillan.

14. Seconde partie, LXVII, p. 589.

15. Le français, assorti dans l'épisode de Roque Guinart d'une variante régionale gasconne, l'italien et, dans la Péninsule, outre le « romance castellano », le catalan, le gitan du polyglotte Ginés de Pasamonte, et le portugais d'une églogue de Camoes.

16. «[...] el primer escalón de las ciencias » (seconde partie, XVI, p. 160). Il s'agit du latin et du grec, étudiés par don Lorenzo à Salamanque. 
17. «[...] son meros romancistas, sin saber otras lenguas ni otras ciencias que adornen y despierten y ayuden a su natural impulso [...]» (seconde partie, XVI, p. 160).

18. «Tengo hasta seis docenas de libros, cuáles de romance y cuáles de latín [...]» (seconde partie, XVI, p. 157).

19. Par exemple dans le passage, antérieur à l'épisode de Barataria, sur l'opposition entre « éructer », " eructar », et " roter », « regoldar» (seconde partie, XLIII, p. 381). Mais, quoi qu'en dise don Quichotte, le premier n'a pas supplanté le second, les deux termes coexistant dans le dictionnaire de la Real Academia.

20. Dont l'une, du bachelier Alonso López, est sanctionnée par un tranchant « No entiendo ese latín» de la part de don Quichotte (première partie, XIX, p. 276). Lequel y va parfois lui aussi d'une citation latine, s'attirant de la part de Sancho Panza un éloquent « No entiendo otra lengua que la mía » (seconde partie, II, p. 47).

21. Cf. seconde partie, XIX, p. 185 : «Tout cela revenait pour les paysans à leur parler en grec ou en jargon [...] » (« Todo esto para los labradores era hablarles en griego o en jerigonza [...] ).

22. Première partie, XXV, p. 355 : «Hélène même n'en approche, et Lucrèce ne l'atteint pas non plus, ni aucune autre [...] des âges passés, grec, barbare, ou romain » («[...] ni la llega Elena, ni la alcanza Lucrecia, ni otra alguna [...] de las edades pretéritas, griega, bárbara o latina »). Remarquer la place centrale de «bárbara ».

23. « $\mathrm{Y}$ con estos latinicos [...] os tendrán siquiera por gramático; que el serlo no es de poca honra y poco provecho el día de hoy » (première partie, Prologue, p. 99). La gramática, le premier des arts du trivium médiéval, correspondant à l'étude de la langue et de la littérature latines, donc à une classification archaïque au XVII ${ }^{\mathrm{e}}$ siècle, on voit que Cervantès rejette les tenants d'une culture pédante. Même chose avec ces mots de don Quichotte à Sancho : «[...] tu n'es pas obligé de savoir le latin, comme certains qui croient le savoir, et n'y entendent rien » (« [...] no estás tú obligado a saber latín, como algunos que presumen que lo saben, y lo ignoran ", seconde partie, XXIX, p. 274).

24. « [...] la lengua, sobre quien tiene poder el vulgo y el uso » (seconde partie, XLIII, p. 381).

25. Et qui désigne parfois aussi la forme poétique ainsi nommée, ou tout simplement un récit, une histoire.

26. Mais le Quichotte ne dit jamais «Castilla » ni « castellano »pour le pays et pour la nationalité, bien sûr, mais "España " et "español ", alors que par les termes "romance castellano", « lenguacastellana » ou « castellano » est maintenu le lien avec l'origine castillane de la langue nationale, le castillan ayant recouvert l'aire linguistique hispanique.

27. "[...] es el deseo tan grande que casi todos tenemos de volver a España, que los más de aquellos (y son muchos) que saben la lengua como yo, se vuelven a ella [...] y dejan allá a sus mujeres y sus hijos... que es dulce el amor de la patria » (seconde partie, LIV, pp. 481-482).

28. «[...] mamé la fe católica en la leche [...]» (seconde partie, LXIII, p.562).

29. «[...] no sabía hablar cristiano » (première partie, XXXVII, p. 513).

30. $C f$. note 11 .

31. Ce possessif s'applique aussi à la patrie - «nuestra España », dit le " cautivo »(première partie, XXXIX, p. 524) - et aux auteurs nationaux, ce qu'attestent les formules «el gran poeta castellano nuestro » et «nuestro poeta » par lesquelles don Quichotte désigne Garcilaso de la Vega (seconde partie, VI, p. 80, et VIII, p. 91).

32. Mais dans quelle langue a-t-il lu Virgile? (seconde partie, XLI, p. 365 : «[...] yo he leído en Virgilio [...]»).

33. Appelé «toscano idioma ", en quoi on voit là aussi un stade d'évolution d'une autre langue romane (seconde partie, LXII, p.553).

34. « [...]lengua del alma » (seconde partie, XVI, p. 161).

35. Première partie, Prologue, p. 95. 
36. Disons que la systématisation des professions de foi en faveur du castillan et la récurrence du phénomène de la traduction, avec cette langue comme point d'aboutissement, sont si frappantes que Cervantès, hostile à l'affectation latiniste, semble nous renvoyer aux temps où le castillan s'était imposé.

37. . L'ami dissuade Cervantès de mendier «[...] maximes de philosophes, paraboles des Saintes Ecritures, fables de poètes, discours de rhétoriciens, miracles de saints [...]» («sentencias de filósofos, consejos de la Divina Escritura, fábulas de poetas, oraciones de retóricos, milagros de santos »), et lui conseille de «[...]faire en sorte que [...] avec des mots expressifs [...] et bien trouvés, votre période acquière sonorité et agrément» («procurar que [...] con palabras significantes [...] bien colocadas, salga vuestra oración y período sonoro y festivo ", première partie, Prologue, p. 101). Noter que, malgré son amour des langues anciennes et des «buenos poetas » dans ces langues, et malgré son « peu d'affection » - « mal cariño » - envers la " poesía de romance », Lorenzo de Miranda compose en castillan sa glose à quatre vers écrits eux aussi en castillan.

38. «[...] reprochador de voquibles [...]», seconde partie, III, p. 56. Mais, même si don Quichotte appelle son écuyer "prevaricador del buen lenguaje " (seconde partie, XIX, p. 188), la «lengua romance » si particulière de Sancho est celle qu'il a « tétée avec le lait », source d'une légitimité qu'il revendique à la même page : «[...] on ne doit pas obliger le natif du Sayago à parler comme un Tolédan » («[...] no hay para qué obligar al sayagués a que hable como el toledano »).

\section{RÉSUMÉS}

Cet article prend pour point de départ le fait que le Quichotte se rattache à une tradition répandue parmi les romans de chevalerie médiévaux, celle d'un présumé original dans une langue plus ancienne et plus prestigieuse que la langue romane. Mais Cervantès va plus loin. En réactivant à sa façon les circuits médiévaux de traduction - en s'intéressant aussi à la traduction contemporaine -, et en observant les spécificités des langues anciennes et modernes, il met en vedette la langue romane et la littérature dans cette langue. Sa langue maternelle s'était certes imposée depuis des siècles, mais par ses professions de foi en faveur des possibilités expressives de cette langue romane perçue comme en constante évolution, par sa méfiance envers certaine affectation latinisante, Cervantès semble vouloir souligner l'amour qu'il porte au castillan.

This article has for starting point the fact that the Quijote is in keeping with a widespread tradition of medieval chivalric romance: the presumed original is presented as written in a language older and more prestigious than Romance. But Cervantes went further. By means of his own reactivation of the medieval channels of translation, of his interest in contemporary translation, and of his attention to the peculiarities of ancient and modern languages, Cervantes put the spotlight on Romance language and literature. His mother tongue had indeed compelled recognition for many centuries, but through his professions of faith in favour of the expressive possibilities of a Romance language seen as in constant evolution, and through his suspicions about a certain kind of Latinizing affectedness, it seems that Cervantes aimed at underlining the love he had for the Castilian language. 
INDEX

Mots-clés : traduction, Don Quichotte, langue romane, Cervantès, langue romane castillane, romans de chevalerie

Keywords : translation, Don Quijote, Romance language, Cervantes, Romance Castilian language, chivalric romance

\section{AUTEUR}

PIERRETTE PELLEN-BARDE

Université de Paris XII -Val de Marne 